\title{
Investigation of Laminar Flow of Fluid with One Porous Bounding Wall
}

\author{
Ramesh Yadav ${ }^{1}$, Manju Agarwal' ${ }^{2}$, Vivek Joseph ${ }^{3}$ \\ ${ }^{1}$ Research Scholar, Uttar Pradesh Technical University Lucknow U P, India \\ ${ }^{2}$ Department of Mathematics \& Astronomy, University of Lucknow, Lucknow U P, India \\ ${ }^{3}$ Department of Mathematics BBDNIIT Lucknow U P, India
}

\begin{abstract}
The numerical solution is used to investigation of laminar flow of fluid with one porous bounding wall. Objective of study is the effect of non-zero tangential slip velocity of, velocity field, pressure gradient and mass transfer. The problem is also studied by using power series method in conjunction with an unconstrained optimization procedure. The solution presented here is valid for much larger Reynolds number compare to earlier investigation.
\end{abstract}

Keyword: Porous medium, Non-Newtonian Fluid, slip coefficient, Reynolds number, Laminar flow

\section{Nomenclature}

$h$ Height of the channel;

$x$ The axial distance from the channel entrance;

$y$ The coordinate axis perpendicular to the channel walls measured from the Non-porous wall;

$u$ Velocity component in the $x$-direction;

$u_{0}$ Average velocity over the channel at channel inlet;

$R e_{i W}$ Wall Reynolds number, $\frac{V_{W} w^{h}}{v}$;

$R e_{\text {ent }}$ Reynolds Number for flow entering the channel, $u 0 \mathrm{~h} / \mathrm{v}$;

v Velocity component in the y- direction;

$\tau_{W}$ Velocity of the fluid through the membrane;

$V \vee / v_{w}:$

\section{Greek Symbols}

$\rho$ Solution density;

$\lambda$ Dimensionless variable in the $y-$ direction, $y / h$;

$\mu$ Viscosity;

$v$ Kinematic viscosity;

$\alpha$ Surface characteristic of the membrane;

$\Delta p \quad$ Dimensionless pressure drop,

$2[p(0, \lambda)-p(x, \lambda)] /\left(\rho u_{0}^{2}\right)$;

$\phi$ Slip coefficient, $\sqrt{k} / \alpha h$;

$\psi$ Stream Function;

\section{Introduction}

The study of flow of fluid through porous media is very prevalent in nature, and therefore the study of flow through a porous medium has become of principle interest in many and engineering applications. The thermal and solute transport by fluid flowing through a porous matrix is phenomenon of great interest from the theory and application point of view. Heat transfer in the case of homogeneous fluid-saturated porous media has been studied with relation of different applications like dynamic of heat underground springs, terrestrial heat flow through aquifer, hot fluid and ignition front displacements in reservoir engineering, heat exchange between soil and atmosphere and heat exchange with fluidized beds. In this assumed viscous fluid by porous walls, fluid passes through in a variety of physical situations such as the control of boundary layer with suction or injection, filtration, membrane separation process and biological transport in living system etc. Many research workers have investigated the steady, incompressible laminar flow of a viscous incompressible fluid through a porous channel and found the solution for a small Reynolds number. Following authors Terrill [11, 12], Robinson W. A. [9], Cox S. M. [5], have extended Berman's Problem and obtained solution for both small Reynolds numbers. But the experimental investigation reveals the existence of slip velocity at the porous bounding surface and is connected with the presence of a thin layer of stream wise moving fluid just below the surface of the porous medium. Beavers and Joseph [2], has studied the mass efflux experiments and proved the existence of a non-zero tangential (slip) velocity on the surface of the permeable boundary. Avrament et al. [1], has studied the Investigation of stability of a laminar flow in a parallel plate channel filled with a fluid saturated porous medium. Bujurke et al. [4], has studied the Expansion and Finite Difference computations of internal laminar Flow Separation. Jafaryar et al. [6], has studied the Analytical investigation of laminar flow through expanding or contracting gaps with porous wall and the solution method is applied the Optimal homotopy asymptotic method(OHAM), in his research the OHAM was successfully applied to find the analytical solution for laminar, isothermal, incompressible and viscous flow in a rectangular domain bounded by two moving porous walls, which enable the fluid to enter or exit during successive expansions or contractions.

N. T. El-Dube et al. [8], has studied the effect of the uniform magnetic field on the peristaltic motion of a non-Newtonian Eyring-Powel fluid through porous medium in a finite horizontal channel. This phenomenon is modulated mathematically by a system of differential equations which describe the continuity and momentum. The system is solved 


\section{International Journal of Science and Research (IJSR) \\ ISSN (Online): 2319-7064}

Index Copernicus Value (2013): 6.14 | Impact Factor (2014): 5.611

analytically under the assumption of long wavelength and low Reynolds number. The velocity, the pressure distribution, the local wall shear stress and the volume flow rate are obtained as functions of the physical parameters of the problem. R. Muthuraj and Srinivas [8], has studied the Influence of magnetic field and wall slip conditions on steady flow between parallel flat wall and a long wavy wall with soret effect and find out that the effect of increasing suction parameter suppresses the velocity while it enhances the fluid temperature, Nusselt number increases with increasing value of Prandtl number and Reynolds number at the flat wall $\mathrm{y}=0$ but this behaviour is reversed at the wavy wall $y=h$. In this paper we have study on the effect of fluid velocity in different slip coefficient and Reynolds Number. And what is effect of fluid velocity in the difference dimensionless coefficient $\lambda$. The effect of fluid flow at a constant dimensionless coefficient $\lambda$ with different slip coefficient and Reynolds number

\section{Mathematical Formulation}

We assume the laminar flow of an incompressible fluid of density $\rho$, kinematic viscosity $v$, the channel is of length $\mathrm{L}$ and Height $h$. The coordinate system of used for the description of the problem is shown in Fig 1. The axial coordinate measured from the channel entrance is denoted by $x$ and $y$ the coordinate axis perpendicular to the channel walls measured from the non-porous wall, the value of $u$ and $v$ represent the velocity component in $x$ and $y-$ directions respectively and $p$ is the pressure.

The basic equation of the Navier-Stokes equations for steady state, two dimensional, incompressible fluid motions under the assumed conditions are

$$
\begin{aligned}
& u \frac{\partial u}{\partial x}+\frac{v}{h} \frac{\partial u}{\partial \lambda}=-\frac{1}{\rho} \frac{\partial p}{\partial x}+v\left(\frac{\partial^{2} u}{\partial x^{2}}+\frac{1}{h^{2}} \frac{\partial^{2} u}{\partial \lambda^{2}}\right)(1) \\
& u \frac{\partial v}{\partial x}+\frac{v}{h} \frac{\partial v}{\partial \lambda}=-\frac{1}{\rho h} \frac{\partial p}{\partial \lambda}+v\left(\frac{\partial^{2} v}{\partial x^{2}}+\frac{1}{h^{2}} \frac{\partial^{2} v}{\partial \lambda^{2}}\right)(2) \\
& \frac{\partial u}{\partial x}+\frac{1}{h} \frac{\partial v}{\partial \lambda}=0 \text { (3) }
\end{aligned}
$$

Boundary conditions are

$u(x, 0)=0, v(x, 0)=0(4)$

$u(x, 1)=v_{w}$ (Constant) $(5)$

$u(x, 1)=-\phi \frac{\partial u}{\partial \lambda}(6)$

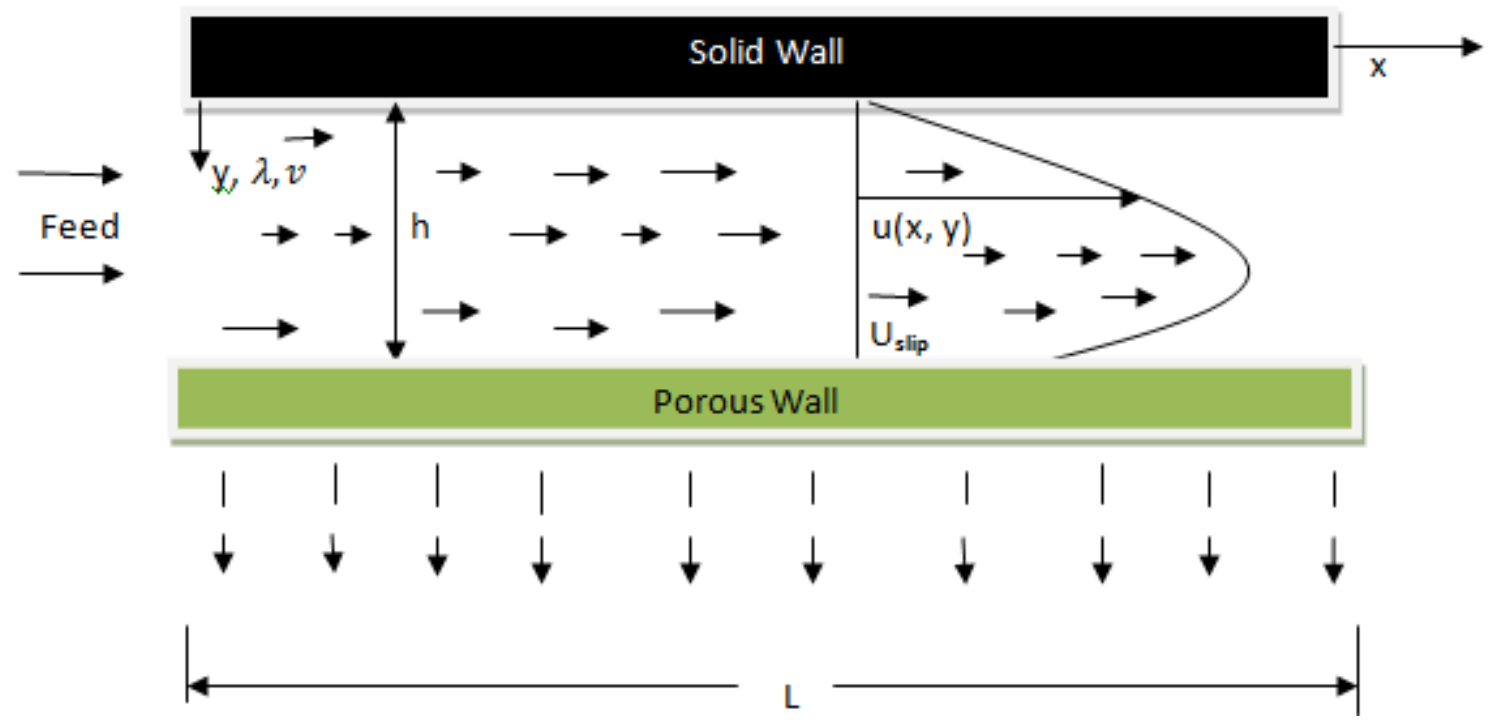

Figure 1: The coordinate system used in the solution of the two dimensional steady

State Navier-Stokes equation in Presence of Magnetic field

Where $\lambda=\frac{y}{h}$ is the dimensionless variable in the $\mathrm{y}-$ direction?

The slip velocity at the membrane surface is proportional to the shear rate at the permeable boundary. The slip velocity is connected with the presence of a thin layer of stream wise moving fluid in the boundary region just below the permeable surface. The fluid in this layer is considered to be pulled along by the flow above the porous surface. $\phi=\sqrt{k} / \alpha h$ is the slip coefficient, where $k$ is the permeability of the membrane material and $\alpha$ is a dimensionless constant dependent on the surface characteristics of the membrane. We introducing the stream function

$$
\left.\begin{array}{c}
u=\frac{\partial \psi}{\partial y}=\frac{\partial \psi}{h \partial \lambda} \\
v=-\frac{\partial \psi}{\partial x}
\end{array}\right\}
$$

A proper stream function (Berman [3]) of the form $\psi(x, \lambda)=\left(h u_{0}-v_{w} x\right) f(\lambda)(8)$

From Navier Stokes equation (1), (2) and eliminating p we get

$$
\begin{gathered}
\frac{\partial}{\partial \lambda}\left(-v_{w}\left(f^{2}\right)^{2}+\frac{v_{w}}{h} f f^{m}-\frac{v}{h^{s}} f^{m}\right)=0 \\
\frac{\partial}{\partial \lambda}\left(\frac{v_{w}}{h}\left(\left(f^{g}\right)^{2}-f f^{v}\right)+\frac{v}{h^{s}} f^{m}\right)=0 \\
\operatorname{Re}\left[f^{v} f^{w}-f f^{m}\right]+f^{I V}=0
\end{gathered}
$$

Equation (12) is one of the Falkner - Skan family of equations.

New set of boundary conditions are

\section{Volume 5 Issue 1, January 2016}




\section{International Journal of Science and Research (IJSR) \\ ISSN (Online): 2319-7064}

Index Copernicus Value (2013): 6.14 | Impact Factor (2014): 5.611

$$
\begin{gathered}
f=0, f^{s}=0 \text { at } \lambda=0 \\
f=1, f^{s}=-\phi f^{\prime \prime} \text { at } \lambda=1
\end{gathered}
$$

\section{Method of Solution}

We use the solution of non linear differential equation by perturbation method

$$
\begin{aligned}
& \text { Let } f(\lambda)=\sum_{n=0}^{\infty} R e^{n} f_{n}(\lambda)(14) \\
& \text { Or } \\
& f(\lambda)=f_{0}(\lambda)+\operatorname{Ref}_{1}(\lambda)+\operatorname{Re}^{2} f_{2}(\lambda)+\operatorname{Re}^{3} f_{3}(\lambda)(15) \\
& f_{n}^{I V}=\sum_{\mathrm{r}=0}^{\mathrm{n}-1}\left[\mathrm{f}_{\mathrm{r}} \mathrm{f}_{\mathrm{n}-1-\mathrm{r}}^{\mathrm{aw}}-\mathrm{f}^{\prime}{ }_{\mathrm{r}} \mathrm{f}_{\mathrm{n}-1-\mathrm{r}}^{\mathrm{w}}\right], \mathrm{n}=1,2,3, \ldots(16) \\
& f_{1}=-\frac{2(1+\phi)^{2}}{35(1+4 \phi)^{2}} \lambda^{7}+\frac{(1+\phi)(1+2 \phi)}{5(1+4 \phi)^{2}} \lambda^{6}-\frac{3(1+2 \phi)^{2}}{10(1+4 \phi)^{2}} \lambda^{5}+\frac{3\left(9+90 \phi+272 \phi^{2}+296 \phi^{\mathrm{a}}\right)}{70(1+4 \phi)^{\mathrm{s}}} \lambda^{3}- \\
& \frac{2\left(4+44 \phi+139 \phi^{\mathrm{2}}+162 \phi^{\mathrm{a}}\right)}{35(1+4 \phi)^{\mathrm{s}}} \lambda^{2} \\
& f_{2}=\frac{4(1+\phi)^{\mathrm{g}}}{5775(1+4 \phi)^{\mathrm{g}}} \lambda^{11}-\frac{2(1+\phi)^{\mathrm{g}}(1+2 \phi)}{525(1+4 \phi)^{\mathrm{g}}} \lambda^{10}+\frac{(1+\phi)(1+2 \phi)^{\mathrm{a}}}{210(1+4 \phi)^{\mathrm{g}}} \lambda^{9}-\frac{3(1+2 \phi)^{\mathrm{g}}}{560(1+4 \phi)^{\mathrm{g}}} \lambda^{8}+ \\
& \frac{3(1+\phi)\left(9+90 \phi+272 \phi^{2}+296 \phi^{\mathrm{a}}\right)}{1225(1+4 \phi)^{4}} \lambda^{7}-\frac{\left(226+2712 \phi+11064 \phi^{2}+19936 \phi^{\mathrm{a}}+13248 \phi^{4}\right)}{4200(1+4 \phi)^{4}} \lambda^{6}+ \\
& \frac{2(1+2 \phi)\left(4+44 \phi+139 \phi^{2}+162 \phi^{\mathrm{a}}\right)}{175(1+4 \phi)^{4}} \lambda^{5}+ \\
& \frac{\left(147831+2965407 \phi+21565566 \phi^{2}+72033732 \phi^{3}+115788432 \phi^{4}+72456480 \phi^{5}\right)}{970200(1+4 \phi)^{5}} \lambda^{3}- \\
& \frac{\left(105173+2103460 \phi+15241240 \phi^{2}+50798240 \phi^{\mathrm{a}}+81732304 \phi^{4}+51280960 \phi^{5}\right)}{646800(1+4 \phi)^{5}} \lambda^{2}(20)
\end{aligned}
$$

Putting these value in equations (17)

We get

$$
\begin{aligned}
& f(\lambda)=-\frac{2(1+\phi)}{(1+4 \phi)} \lambda^{3}+\frac{3(1+2 \phi)}{(1+4 \phi)} \lambda^{2}+ \\
& \operatorname{Re}\left[-\frac{2(1+\phi)^{2}}{35(1+4 \phi)^{2}} \lambda^{7}+\frac{(1+\phi)(1+2 \phi)}{5(1+4 \phi)^{2}} \lambda^{6}-\frac{3(1+2 \phi)^{2}}{10(1+4 \phi)^{2}} \lambda^{5}+\frac{3\left(9+90 \phi+272 \phi^{2}+296 \phi^{\mathrm{a}}\right)}{70(1+4 \phi)^{\mathrm{a}}} \lambda^{3}-\frac{2\left(4+44 \phi+139 \phi^{2}+162 \phi^{\mathrm{s}}\right)}{35(1+4 \phi)^{\mathrm{s}}} \lambda^{2}\right] \\
& +\operatorname{Re}^{2}\left[\frac{4(1+\phi)^{\mathrm{s}}}{5775(1+4 \phi)^{\mathrm{a}}} \lambda^{11}-\frac{2(1+\phi)^{2}(1+2 \phi)}{525(1+4 \phi)^{\mathrm{s}}} \lambda^{10}+\frac{(1+\phi)(1+2 \phi)^{\mathrm{a}}}{210(1+4 \phi)^{\mathrm{a}}} \lambda^{9}-\frac{3(1+2 \phi)^{\mathrm{s}}}{560(1+4 \phi)^{\mathrm{a}}} \lambda^{8}+\frac{3(1+\phi)\left(9+90 \phi+272 \phi^{2}+296 \phi^{\mathrm{s}}\right)}{1225(1+4 \phi)^{4}} \lambda^{7}-\right. \\
& \frac{\left(226+2712 \phi+11064 \phi^{2}+19936 \phi^{\mathrm{a}}+13248 \phi^{4}\right)}{4200(1+4 \phi)^{4}} \lambda^{6}+\frac{2(1+2 \phi)\left(4+44 \phi+139 \phi^{2}+162 \phi^{\mathrm{a}}\right)}{175(1+4 \phi)^{4}} \lambda^{5}+ \\
& \frac{\left(147831+2965407 \phi+21565566 \phi^{2}+72033732 \phi^{3}+115788432 \phi^{4}+72456480 \phi^{5}\right)}{970200(1+4 \phi)^{5}} \lambda^{3}- \\
& \left.\frac{\left(105173+2103460 \phi+15241240 \phi^{2}+50798240 \phi^{\mathrm{g}}+81732304 \phi^{4}+51280960 \phi^{5}\right)}{646800(1+4 \phi)^{5}} \lambda^{2}\right]
\end{aligned}
$$

\section{Result and Discussion}

In this we study the laminar flow of fluid with porous bounding walls. One of the walls being porous with velocity slip at porous medium is studied using computer extended series and is also by using power series method. The motion of the fluid is governed by a non-linear ordinary differential equation (11) with boundary condition (12) \& (13). In this paper we study the variation of slip coefficient $\phi$, variation of Reynolds No $R e$, variation of width $\lambda$ in different constant. 


\section{International Journal of Science and Research (IJSR) \\ ISSN (Online): 2319-7064 \\ Index Copernicus Value (2013): 6.14 | Impact Factor (2014): 5.611}

The variation of slip coefficient of the walls increases the velocity of fluid slowly increases and then sharply decreases at constant Reynolds number. which is show in the Fig. 2.a,

b \& c. The variation of Reynolds number is shown in Fig. 3.

a $\&$ b, which show that when we increase the Reynolds number at constant slip coefficient, the velocity of fluid is slowly increases and then sharply decreases.

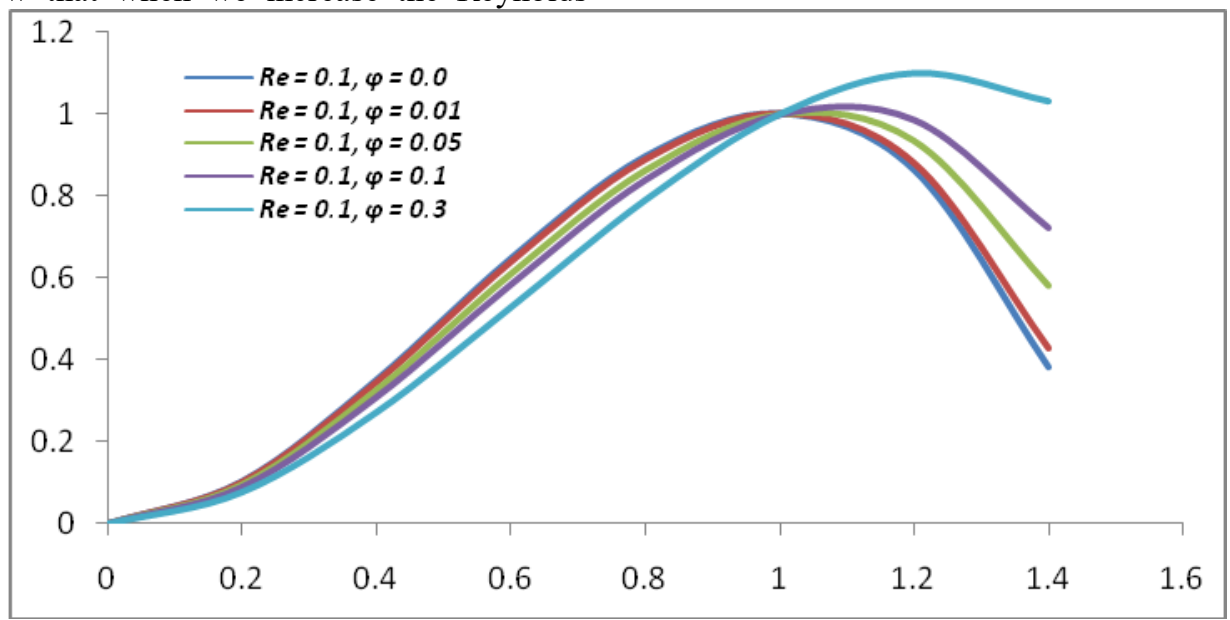

(a)

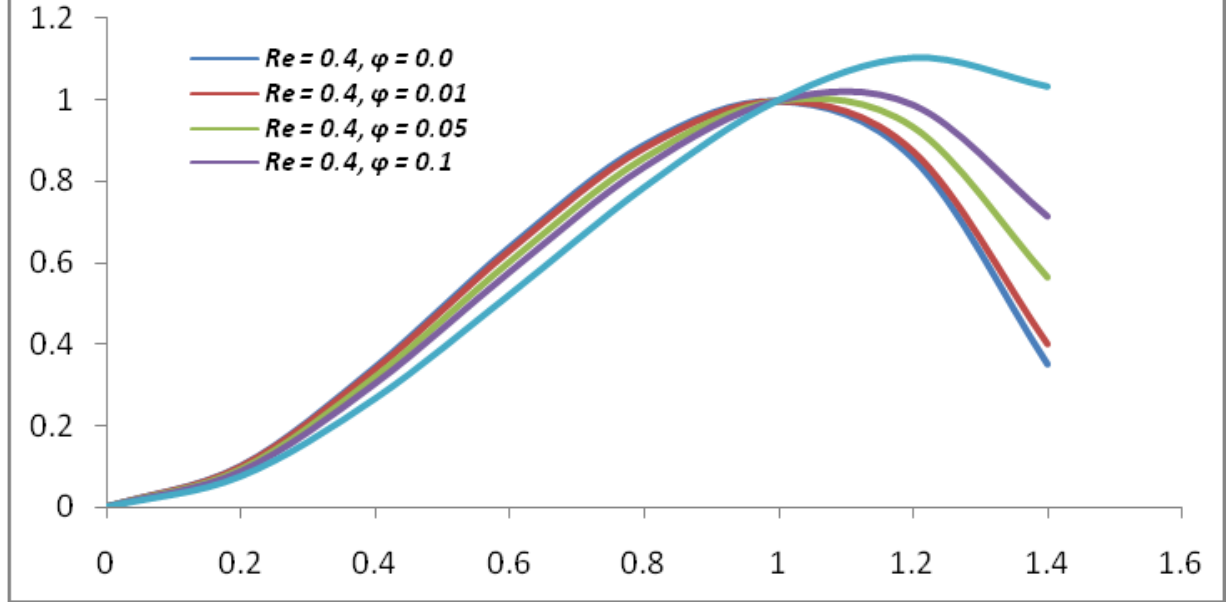

(b)

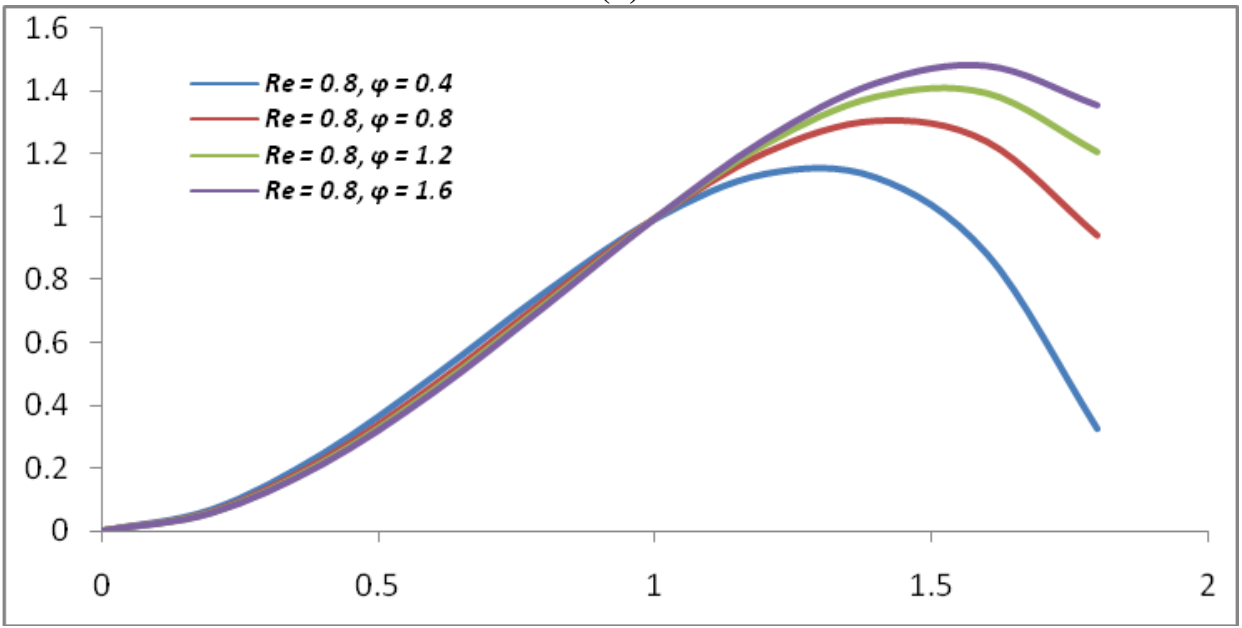

(c)

Figure 2: Graph between the dimensionless variable $\lambda$ and velocity of the fluid $f(\lambda)$ 
International Journal of Science and Research (IJSR)

ISSN (Online): 2319-7064

Index Copernicus Value (2013): 6.14 | Impact Factor (2014): 5.611

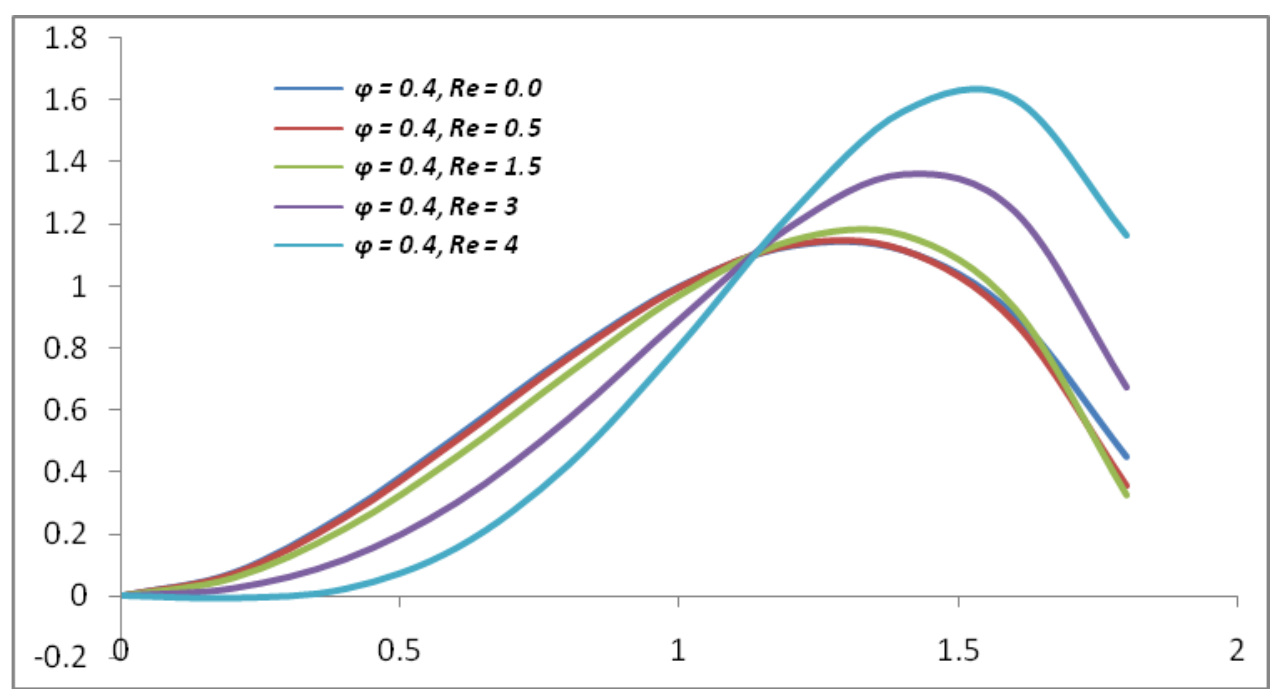

(a)

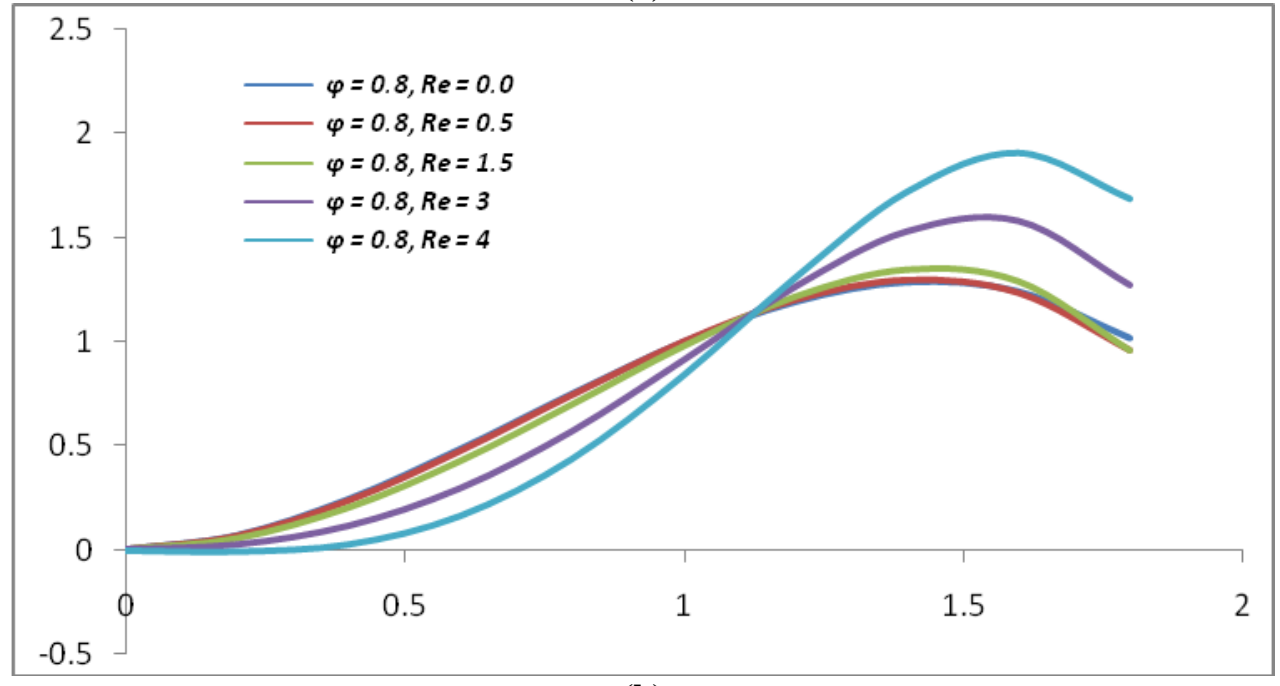

(b)

Figure 3: Graph between the dimensionless variable $\lambda$ and velocity of the fluid $f(\lambda)$

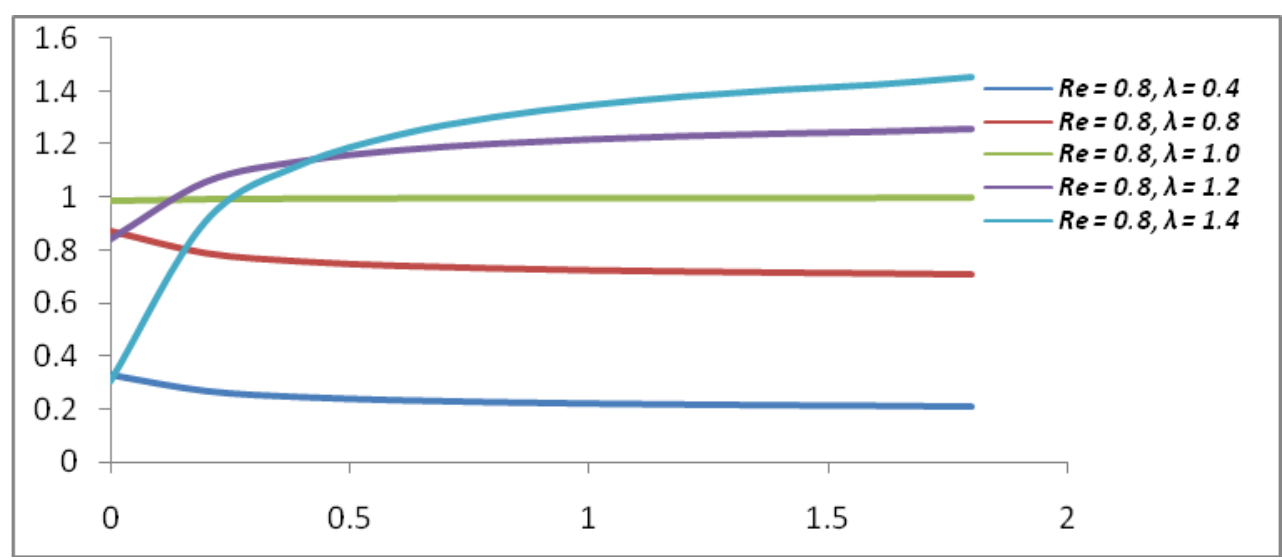

Figure 4: Graph between slip coefficient $\phi$ and velocity of the fluid $f(\lambda)$

Volume 5 Issue 1, January 2016 
International Journal of Science and Research (IJSR)

ISSN (Online): 2319-7064

Index Copernicus Value (2013): 6.14 | Impact Factor (2014): 5.611

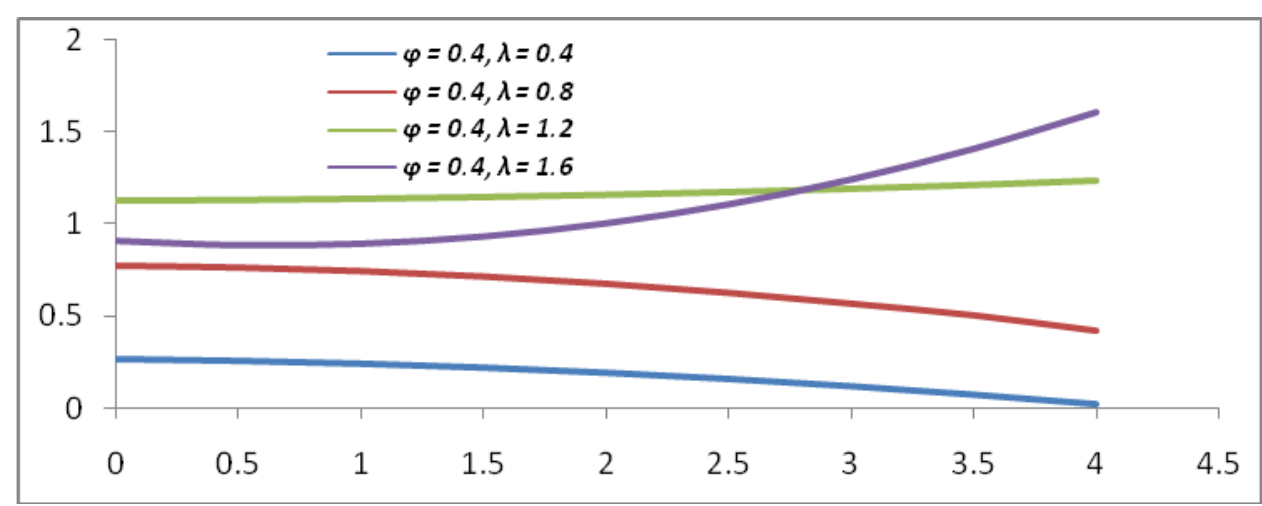

(a)

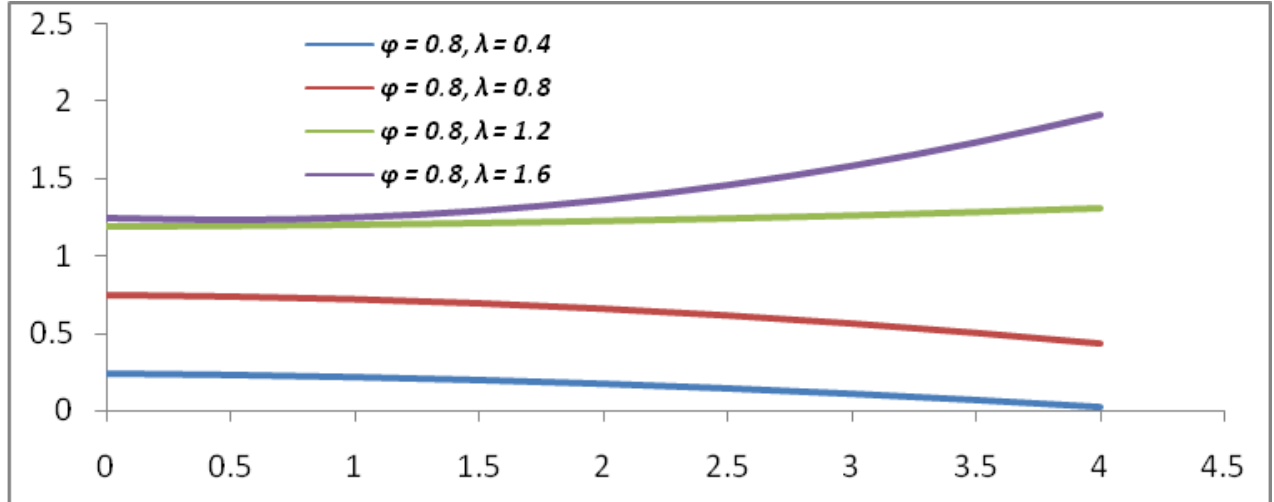

(b)

Figure 5: Graph between Reynolds No. Re and velocity of the fluid $f(\lambda)$

The variation of dimensionless variable at constant Reynolds No, we see that increase of slip coefficient, the velocity of fluid show dual characters when dimensionless variable is less than unity, the velocity of the fluid is slowly decreases and then form the constant velocity whereas when the dimensionless variable is greater than unity the velocity of the fluid is slowly increases and then form the constant velocity. It is shown in the Fig 4. Now we see the Fig. 5a \& $\mathrm{b}$, which is show that the variation of dimensionless variable at constant slip coefficient, when we dimensionless variable, we see that the velocity of fluid show also dual characters first when the dimensionless variable is less than unity then the velocity of the fluid is continuously slowly-slowly decreases, secondly when the dimensionless variable is greater than unity then the velocity of fluid is continuously slowly-slowly increases.

\section{Conclusion}

In this paper, numerical technique method is applied to solve the problem of non-Newtonian fluid flow in porous bounding wall. The results show that the increment of slip coefficient, the effect of velocity component both of them increases with increase of slip coefficient and increment in Reynolds number has similar effect on velocity components both of them increases with increase of Reynolds number. At higher Reynolds number the maximum velocity point is shift to the solid wall where shear stress becomes larger as the Reynolds number grows.

\section{References}

[1] A.A. Avrament, A.V.Kuznetsav , B.I.Nasak and D.G.Blimov (2005); "Investigation of stability of a laminar flow in a parallel plate channel filled with a fluid saturated porous medium." Jour of Physics of fluids Vol. 17 pp. 094102-1 to 094102-6.

[2] Beavers G. S., and Joseph D. D., [1967]; "Boundary conditions a a Naturally Permeable wall" Jour. Of Fluid Mech. Vol. 30, pp. $197-207$.

[3] Berman A. S., (1953); "Laminar Flow in channels with porous walls.” Jour. Appl. Phys. Vol. 24, pp. 1232 1235 .

[4] Bujurke N. M., Pedley T. J., and Tutty O. R., [1996]; "Comparison of Series Expansion and Finite Difference computations of Internal laminar Flow Separation.” Phil. Trans. Roy. Soc. Lond. A453, pp. $1751-1773$.

[5] Cox, S. M. (1991); "Analysis of Steady Flow in a Channel with One Porous Wall or with Accelerating Walls." SIAM Jour. Appl. Math., Vol. 51, No. 2, pp. $429-438$.

[6] M. Jafaryar, F. Farkhadia , E. Mohammadian, M. Hosseini and A. M. Khazaee (2014); "Analytical investigation of laminar flow through expanding or contracting gaps with porous walls." Propulsion and Power Research Vol. 3 (4), pp. 222 - 229.

[7] N. T. E1-Dabe, G. Ismail, F. O. Darwesh (2013); "Peristaltic transport of a magneto non-Newtonian fluid through a porous medium in a horizontal finite channel." IOSR. Journal of Mathematics (IOSR-JM) e-ISSN: 2278-3008, PISSN: 2319 -7676, Vol. 8, issue 6, pp. $32-$ 39.

[8] R. Muthuraj and S. Srinivas (2009); ,Influence of Magnetic field and wall slip conditions on steady flow between parallel flat wall and a long wavy wall with soret effect." Jour. Of Naval Architecture and Marine Engineering, Vol. 6 Issue. 2, pp. $62-71$. 


\section{International Journal of Science and Research (IJSR) \\ ISSN (Online): 2319-7064}

Index Copernicus Value (2013): 6.14 | Impact Factor (2014): 5.611

[9] Robinson, W. A. (1976); "The Existence of Multiple Solutions for the Laminar Flow in a Uniformly Porous Channel with Suction at Both Walls." Jour. Eng. Math., Vol. 10, pp. $33-40$.

[10] S. Ganesh and S. Krishnambal (2006); "Magneto hydrodynamics Flow of Viscous fluid between two parallel plates." Jour. Of Applied Sciences 6(11) pp. $2420-2425$.

[11] Terrill, R. M. (1964); "Laminar Flow in a Uniformly Porous Channel.” Aeronaut. Quart.. Vol. 15, pp. 299 310.

[12] Terrill, R. M. (1965); "Laminar Flow through a channel with uniformly Porous Walls of Different Permeability." Appl. Sci. Res., Sec. A, Vol. 15, pp. $440-468$. 